\title{
Lessons Learned from an Interaction-Kiosk for Open Selection of Input Devices of a Gaming Application
}

\author{
Andreas Lorenz \\ Ferry Pramudianto \\ Andreas Zimmermann \\ Fraunhofer Institute for Applied Information Technology \\ Schloss Birlinghoven \\ 53754 St. Augustin, Germany \\ +49 (0) 2241/14 \{-2971, -2421, -2561$\}$ \\ \{andreas.lorenz, ferry.pramudianto, andreas.zimmermann\}@fit.fraunhofer.de
}

\begin{abstract}
Traditional computer applications understand mouse and keyboard input for controlling the behavior of the system. Depending on the goal, task and situation of the user, other input devices can be more appropriate to meet the user's personal attributes. To demonstrate the value of enabling the user to select an input device according to personal preferences, this work combines a device independent control mechanism with a game application. The paper illustrates seven lessons learned from observing users in playing the game with six devices that feature different modalities.
\end{abstract}

\section{Categories and Subject Descriptors}

H.5.2 [Information Interfaces and Presentation]: User Interfaces - Input devices and strategies, Interaction styles

\section{General Terms}

Design, Experimentation, Human Factors, Verification.

\section{Keywords}

Input devices, remote user interface.

\section{INTRODUCTION}

By principle, software applications react on input commands independently of the device being used to create it. More important than the concrete device is to distinctly define the meaning of a command, and that the user is able to anticipate the triggered reaction of the computer application. The goal is to enable the user to test any input device with an application. In particular the use of devices the user is already familiar with could be transferred and tested in additional or new services. In addition, the use of different interaction styles like gestures and spoken commands are more convenient for some applications.

Eustice et al. [2] envisioned that users should have the freedom to select from a wide range of devices depending on the situation or preference. Iftode et al. [3] identified the need for a simple, universal solution to control different applications in the environment of the user, which end-users are likely to accept easily. Many studies in smart home environments have proved that users can easily interact with their context using handheld devices. Nichols [7] presented positive results after performing an exhaustive study of the efficiency of users using handheld devices to remotely control a stereo and a telephone/digital answering

Copyright is held by the author/owner(s). MobileHCI'09, September 15 - 18, 2009, Bonn, Germany. ACM 978-1-60558-281-8. machine. Some authors introduce mobile phones as the user's favorite device for remote controlling [7]. Using the Personal Universal Controller [6], a user can also speak the name of a command through which this is executed by the system.

Gestures play an important role in human communication. People use gestures as an extension of spoken languages, gestures are sometimes used to communicate when the languages are not compatible anymore. Head-tracker solutions like [4] are designed to work with gestures for replacing traditional pointing devices. Using a Web-cam, it allows users to point and click by simply aiming their face. A combination of pointer position and keystroke input device is described in [1], using miniature video cameras that track finger position where the user can type or point in the air.

\section{THE INTERACTION-KIOSK}

Similar to a kiosk of interaction styles, the Interaction-Kiosk comprises different input devices which provide a shortcut for the users to execute an activity in the application, which normally would be hard to achieve with traditional keyboard and mouse interaction from a distance. The architectural design follows the approach of Virtual Input Devices [5]. Users of the kiosk had the opportunity to select any of the following six realizations of user interfaces: Navigation buttons of an infrared remote control, dragging on the touch-sensitive display of a PDA (by finger or stick), XBox-Controller, Wii-Controller, dancing mat, and camera-based gesture recognition with retro-reflecting marker.
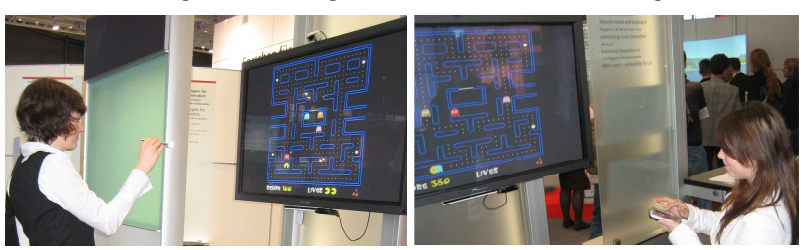

Figure 1 Example of aiming gestures in the air (left) and drawing gestures on the PDA's screen (right)

For the demonstrator, a PacMan game runs in full screen mode. The user's task was to play the game with the chosen device, i.e. to control the small yellow icon moving in the four directions "Up", "Down", "Left" and "Right". On own decision, the user was able to select any other device at any time without interruption of the game.

The left image of Figure 1 shows a user employing a stick for controlling the game. The user is aiming with the stick in the air for making the PacMan moving to the intended direction. The right image of Figure 1 shows another user who is doing similar 
gestures on the screen of the PDA. By dragging with a pen or the finger on the display, the user controls the PacMan as if drawing a line to the intended direction.

\section{LESSONS LEARNED}

To collect feedback, 42 users were interviewed after playing the game. On average, each person used four different devices. People most often choose the dancing mat or the Wii-controller first. Other devices rarely served as first choice. As reason for selecting the particular device first, 24 persons stated their interest in this device. The personal prediction whether a device is useful or not did not have effect on the selection. It seems that people either selected a new device out of curiosity, or a known device they are familiar with.

Lesson learned (1): Spontaneous interest was a trigger for selection of the first device. The shape and predicted performance of a device play a minor role in the selection.

The overall interest was dedicated to the gesture tools (hand gesture, Wii, and dragging on PDA) and the dancing mat. People who explicitly stated "was interested in" as trigger most often choose the dancing mat.

Lesson learned (2): People are interested in innovative interaction styles and do not hesitate to try.

The Xbox-controller was the best input device to the PacMangame. The similar interface of the remote control was second this input style of four arrow keys reached more personal high scores than all other styles together. It is obvious, that the Xboxcontroller is pretty well designed for gaming.

Lesson learned (3): The applied device has impact on user performance. Some devices fit better for performing special tasks than others.

The Xbox-controller was most often assigned with the highest personal rank. The dancing mat, which was most often first choice but last place for personal high-scores, is far behind the top in the personal rankings. Though the first selection was driven by visual attraction and spontaneous interest, the rankings relate to the usefulness of the device for the task.

Lesson learned (4): After people satisfy curiosity and enthusiasm, they prefer useful input devices according to the task.

When asked to assign school grades from 1 ("easy to use") to 5 ("unhandy") to each device, two groups are distinguishable: the Xbox-controller and the remote control with averages below 2, and all other devices with averages above 3 . Even the worst grade for Xbox and remote control are lower than all other averages.

Lesson learned (5): The relevant indicator for the preferred device seems to be the easiness of using the input method.

All methods using gestures were intuitively used by all users, but creating a concrete event needs training of acceleration, speed, and length of movement. From only seven written comments, five were asking for improvements of the gesture tools, in particular the Wii-controller.

Lesson learned (6): Not only the interaction style needs to be intuitive, but the concrete implementation of the interaction device needs to be carefully performed.
People did not hesitate at all in interchanging devices. If a device seemed not to work well, it got replaced by another one. If the device was considered to be boring, another device was employed. Independent of age and sex, users interchanged devices in any order.

Lesson learned (7): It is a natural thing to interchange between devices for remotely controlling a computer application.

\section{SUMMARY}

The paper presented a study on how users select an interaction device for the control of a simple PacMan game application from a distance. In order to achieve this task the users were allowed to take any device from a heterogeneous set of devices with different levels of familiarity to the users.

The study revealed that in the phase of "first contact" the users primarily showed considerable interest in devices tending to be unusual for the use in the specific situation of playing a game. Disregarding the shape and the intended usage of specific devices, most of the users satisfied their curiosity at first and started using uncommon means of interaction. During the second phase the users exchanged the devices with another in a natural way trying to find the one that meets best the requirements of their current situation. The third phase entailed a type of consolidation, in which the users continued using input devices that allowed them to perform the task with the best possible result, i.e. reaching a new high-score.

\section{ACKNOWLEDGMENTS}

This research was supported by the European Commission within the InterMedia Network of Excellence (project No. 038419).

\section{REFERENCES}

[1] Ahmad, F. and Musilek, P. 2006. A keystroke and pointer control input interface for wearable computers. In Proceedings of PerCom 2006, 2-11.

[2] Eustice, K., Lehman, T., Morales, A., Munson, M., Edlund, S. and Guillen, M. 1999. A universal information appliance. IBM Systems Journal, 38(4):575-601.

[3] Iftode, J., Borcea, C., Ravi, N., Kang, P. and Zhou, P. 2004. Smart phone: An embedded system for universal interactions. In Proceedings of FTDCS 2004.

[4] Kjeldsen. R. 2006. Improvements in vision-based pointer control. In Proceedings of Assets 2006, 189-196. ACM.

[5] Lorenz, A., Eisenhauer, M. and Zimmermann, A. 2008. Elaborating a framework for open human computer interaction with ambient services. In Proceedings of PERMID 2008, 171-174.

[6] Myers, B.A. 2002. Mobile devices for control. In Proceedings of Mobile HCI 2002, 1-8, Springer-Verlag.

[7] Nichols, J. 2001. Using handhelds as controls for everyday appliances: A paper prototype study. In Extended Abstracts of CHI 2001, 443-444.

[8] Nichols, J., Myers, B.A., Higgins, M., Hughes, J., Harris, T.K., Rosenfeld, R. and Pignol, M. 2002. Generating remote control interfaces for complex appliances. In Proceedings of UIST 2002. 